\title{
VALORACIÓN DE LA ESCALA NEWS (NATIONAL EARLY WARNING SCORE) COMO PREDICTOR DE SEPSIS EN PACIENTES CON SÍNDROME FEBRIL. SERVICIO MEDICINA INTERNA, HOSPITAL SALVADOR BIENVENIDO GAUTIER, AGOSTO-DICIEMBRE 2019
}

\section{News (National Early Warning Score) scale assessment as sepsis predictor in patients with febril syndrome. Internal Medicine Emergency Department, Salvador Bienvenido Gautier Hospital, August-December 2019}

\section{Angel Díaz ${ }^{\mathrm{a}}$, Mariella Cedano ${ }^{\mathrm{b}}$, Clevy Pérezc $^{\mathrm{c}}$, Yaritza López ${ }^{\mathrm{d}}$, José De Frías Salomón ${ }^{\mathrm{e}}$ y Pedro Olmo ${ }^{f}$}

Recibido: 12 de mayo, $2021 \bullet$ Aprobado: 23 de agosto, 2021

Cómo citar: Díaz A, Cedano M, Pérez C, López Y, De Frías Salomón J, Olmo P. Valoración de la Escala News (National Early Warning Score) como predictor de sepsis en pacientes con síndrome febril. Servicio Medicina Interna, Hospital Salvador Bienvenido Gautier, agosto-diciembre 2019. cysa [Internet]. [citado 22 de febrero de 2022];6(1):45-53. Disponible en: https://revistas.intec.edu.do/index. php/cisa/article/view/2406

\section{Resumen}

Introducción: la utilización de la escala NEWS podría construir una herramienta en el diagnóstico, pronóstico y estratificación precoz ante la sospecha de sepsis. El propósito de esta investigación fue determinar la eficacia de la escala NEWS como predictor de sepsis en pacientes ingresados por síndrome febril en el Hospital Salvador Bienvenido Gautier, en el período de octubre-diciembre 2019.

Material y método: se realizó un estudio descriptivo, prospectivo y de corte transversal donde la técnica utilizada para la recolección de la información fue la evaluación directa de los 43 pacientes admitidos aplicando la escala NEWS y revisión de expedientes clínicos.

Resultados: se determinó que el punto de corte $\geq 6$ en la escala NEWS obtuvo valores de sensibilidad (91\%), especificidad (70\%), índice de Youden de 0.61 , valor predictivo

\footnotetext{
a Universidad Autónoma de Santo Domingo (UASD). Escuela de Medicina. Santo Domingo, República Dominicana.

ORCID: 0000-0003-4197-7929, Correo-e: adiaz36@uasd.edu.do

b Universidad Autónoma de Santo Domingo (UASD).

ORCID: 0000-0001-7446-7357, Correo-e: mcedano31@uasd.edu.do

${ }^{\mathrm{c}}$ Universidad Autónoma de Santo Domingo (UASD).

ORCID: 0000-0001-5396-1815, Correo-e: clevyperez@gmail.com
}

\begin{abstract}
Introduction: The use of the NEWS could build a tool in the diagnosis, prognosis and early stratification in case of suspected sepsis. The purpose of this research was to determine the efficacy of the NEWS as a predictor of sepsis in patients admitted for febrile syndrome at the Salvador Bienvenido Gautier Hospital, October-December 2019.

Materials and methods: A descriptive, prospective and crosssectional study was conducted where the technique used for the collection of the information was the direct evaluation of the 43 admitted patients applying the NEWS and the review of clinical records.

Results: It was determined that the cutoff point $\geq 6$ on the NEWS obtained sensitivity value (91\%), specificity (70\%), Youden index of 0.61 , positive predictive value of $78 \%$, negative predictive value of $88 \%$, ratio of positive likelihood

\footnotetext{
d Universidad Autónoma de Santo Domingo (UASD). ORCID: 0000-0002-3605-7901, Correo-e: yaritzalopez20@gmail.com

e Universidad Autónoma de Santo Domingo (UASD). ORCID: 0000-0003-1971-4196

Correo-e: josedefriassalomon@hotmail.com

${ }_{\mathrm{f}}^{\mathrm{f}}$ Universidad Autónoma de Santo Domingo (UASD).

ORCID: 0000-0002-9617-9476, Correo-e: pedroolmos0707@gmail.com
} 
positivo de $78 \%$, valor predictivo negativo de $88 \%$, razón de verosimilitud positiva de 3 y negativa de 0.1 . La aplicación de la escala NEWS en el sexo masculino presentó una sensibilidad de $92.9 \%$ y especificidad de $87.5 \%$. En los grupos de 18-30 y 71-80 ańos, esta escala presentó una sensibilidad y especificidad de $100 \%$. Los pacientes clasificados como riesgo bajo, moderado y alto fueron diagnosticados con sepsis el $16.67 \%, 63.64 \%$ y un $70 \%$, respectivamente.

Conclusiones: una puntuación mayor o igual a 6 en la escala NEWS se presenta como un predictor estadísticamente significativo que ayudaría en el diagnóstico temprano de sepsis, siendo más sensible que especifica. Fue mejor predictor en el sexo masculino y en los grupos etarios de 18-30 y 71-80 años.

Palabras clave: Escala News; sepsis; síndrome febril; predictor.

\section{Introducción}

La sepsis se define como la disfunción orgánica potencialmente mortal causada por una respuesta desregulada del huésped a la infección. ${ }^{1}$ No es indispensable la invasión de microorganismos en la circulación, pues la inflamación local también desencadena disfunción orgánica a distancia e hipotensión. ${ }^{2}$ Sin embargo, se define shock séptico como una subcategoría de la sepsis en la que las alteraciones circulatorias y del metabolismo celular son lo suficientemente marcadas como para incrementar considerablemente la mortalidad. ${ }^{3}$

En la actualidad, la sepsis representa un problema de salud a nivel mundial, ocupando aproximadamente $2 \%$ de todas las hospitalizaciones en países desarrollados, 6 a $30 \%$ de los pacientes en unidad de cuidados intensivos y una mortalidad que ronda entre el 28-56 \%. ${ }^{4} \mathrm{La}$ incidencia de sepsis ha ido aumentando, esto se le atribuye a la combinación de múltiples factores, como la edad avanzada de los pacientes, el aumento en número y complejidad de procedimientos diagnósticos y terapéuticos, el mayor uso de drogas inmunosupresoras y el creciente número de infecciones por bacterias of 3 and negative of 0.1 . The application of the NEWS scale in males presented a sensitivity of $92.9 \%$ and specificity of $87.5 \%$. In the groups of 18-30 and 71-80 years, this scale presented a sensitivity and specificity of $100 \%$. Patients dissified as low moderate and high risk we high risk were diagnosed with sepsis $16.67 \%, 63.64 \%$ and $70 \%$ respectively.

Conclusions: A score greater than or equal to 6 on the NEWS is presented as a statistically significant predictor that would help in the early diagnosis of sepsis being more sensitive than specific. It was a better predictor in the male sex and in the age groups of 18-30 and 71-80 years

Keywords: NEWS; sepsis; febrile syndrome; predictor; infection.

multiresistentes. ${ }^{5}$ Aunque puede afectar a pacientes anteriormente sanos, lo más común es que ocurra en personas con comorbilidades. ${ }^{6}$

Cada hora de retraso del diagnóstico se asocia con una reducción del $7 \%$ en la supervivencia atribuible a la falta de reconocimiento durante la admisión del paciente. Estas estadísticas preocupantes han motivado en los últimos ańos varios estudios que aporten a los clínicos una escala para su reconocimiento y manejo precoz, dentro del cual se encuentra la escala NEWS, una escala de advertencia que se considera precisa para predecir la mortalidad en pacientes con sospecha de infección que se presentan en el servicio de emergencia.

El National Early Warning Score (NEWS) fue concebida como una escala de reconocimiento temprano y de respuesta al deterioro agudo de los pacientes, cuyo objetivo principal es de estandarizar el proceso de registro, puntuación y respuesta frente a los cambios en los parámetros fisiológicos que se encuentran alterados en el paciente en estado crítico, los cuales son medidos de forma rutinaria. ${ }^{8}$ Se ha determinado que el sistema de puntación NEWS es considerado más preciso para la identificación precoz de sepsis en el departamento de emergencias en comparación con SIRS y qSOFA. ${ }^{9}$ Además, se considera más acertado para predecir la mortalidad dentro de 10-30 días en pacientes que acuden al departamento de emergencia con sepsis sospechada. ${ }^{10}$

\section{Material y métodos}

Se realizó un estudio descriptivo, prospectivo y de corte transversal con el objetivo de determinar la eficacia de la escala NEWS como predictor de sepsis en pacientes ingresados con diagnóstico de síndrome febril en el servicio de Medicina Interna del Hospital Salvador Bienvenido Gautier. Se incluyeron todos los pacientes con fiebre termometrada por encima de 38.1 grados Celsius, con un foco infeccioso identificado y que acudieron al centro durante el periodo octubre-diciembre 2019. Se recogieron las siguientes variables: edad, sexo, grado de fiebre, diagnóstico de ingreso, comorbilidades, valor NEWS y evolución clínica.

La técnica utilizada para la recolección de la información fue la elaboración de un formulario, el cua fue cumplimentado con los datos contenidos en los

Tabla1. Frecuencia de síndrome febril según edad y sexo

\begin{tabular}{|c|c|c|c|c|c|c|}
\hline \multicolumn{7}{|c|}{ Sexo } \\
\hline Grupo etario & \multicolumn{2}{|c|}{ Masculino } & \multicolumn{2}{|c|}{ Femenino } & \multicolumn{2}{c|}{ Total } \\
\hline & No. & $\%$ & No. & $\%$ & No. & $\%$ \\
\hline $18-30$ & 3 & $13.64 \%$ & 3 & $14.29 \%$ & 6 & $13.95 \%$ \\
\hline $31-40$ & 4 & $18.18 \%$ & 3 & $14.29 \%$ & 7 & $16.28 \%$ \\
\hline $41-50$ & 3 & $13.64 \%$ & 5 & $23.81 \%$ & 8 & $18.60 \%$ \\
\hline $51-60$ & 0 & $0.00 \%$ & 4 & $19.05 \%$ & 4 & $9.30 \%$ \\
\hline $61-70$ & 6 & $27.27 \%$ & 3 & $14.29 \%$ & 9 & $20.93 \%$ \\
\hline $71-80$ & 4 & $18.18 \%$ & 0 & $0.00 \%$ & 4 & $9.30 \%$ \\
\hline$>80$ & 2 & $9.09 \%$ & 3 & $14.29 \%$ & 5 & $11.63 \%$ \\
\hline Total & 22 & $100.00 \%$ & 21 & $100.00 \%$ & 43 & $100.00 \%$ \\
\hline
\end{tabular}

Fuente: formularios completados con los expedientes clínicos de los pacientes. expedientes clínicos de los pacientes. Además, se aplicó a escala NEWS, que toma en cuenta la frecuencia respiratoria, saturación de oxígeno, utilización de oxígeno suplementario, temperatura, presión arterial sistólica, frecuencia cardíaca y nivel de conciencia.

Los formularios fueron completados en el momento del alta o fallecimiento con el resultado del examen clínico y pruebas de laboratorio según conste en el expediente clínico. Luego de recolectar la información, los datos fueron procesados mediante los programas, Epi Info ${ }^{\mathrm{TM}}$ versión 7.2.3.0. y Microsoft Excel 2016 en lenguaje español.

\section{Resultados}

En una muestra de 43 pacientes con síndrome febril, se determinó que el sexo masculino fue predominante con $51.16 \%$ de todos los casos. El grupo etario más frecuente con síndrome febril en el sexo masculino fue de 61-70 años, representando un $27.27 \%$, mientras que en el sexo femenino fue el rango de $41-50$ ańos, con un $23.81 \%$ de los casos.

Tomando en cuenta ambos sexos, el grupo etario más afectado fue de 61-70 años, con un $20.93 \%$ de todos los casos (véase tabla 1). 
De 23 pacientes que fueron diagnosticados con \% fallecieron y $66.67 \%$ se encontraban en edad sepsis, 12 presentaron temperatura axilar entre por encima de 61 ańos. El $39 \%$ presentó tempera38.6-39. $0^{\circ} \mathrm{C}$, representando el $52 \%$. De estos, el tura por encima de $39.1{ }^{\circ} \mathrm{C}$, y el $9 \%$ restante entre $41.67 \%$ fueron ingresados bajo el diagnóstico de $38.1-38.5^{\circ} \mathrm{C}$. (véase figura 1 ).

NAC, $25 \%$ con el diagnóstico de pie diabético, 50

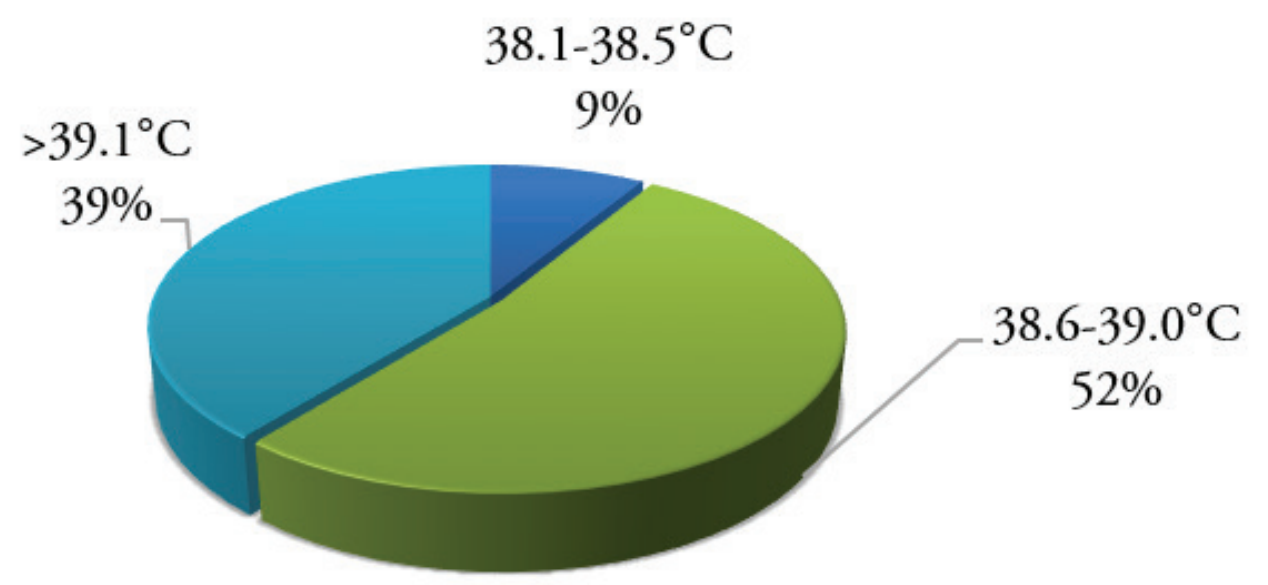

Figura 1. Grado de fiebre en pacientes diagnosticados con sepsis en el servicio de emergencia de Medicina Interna, Hospital Salvador Bienvenido Gautier, agosto-diciembre 2019

Fuente: formularios completados con los expedientes clínicos de los pacientes.

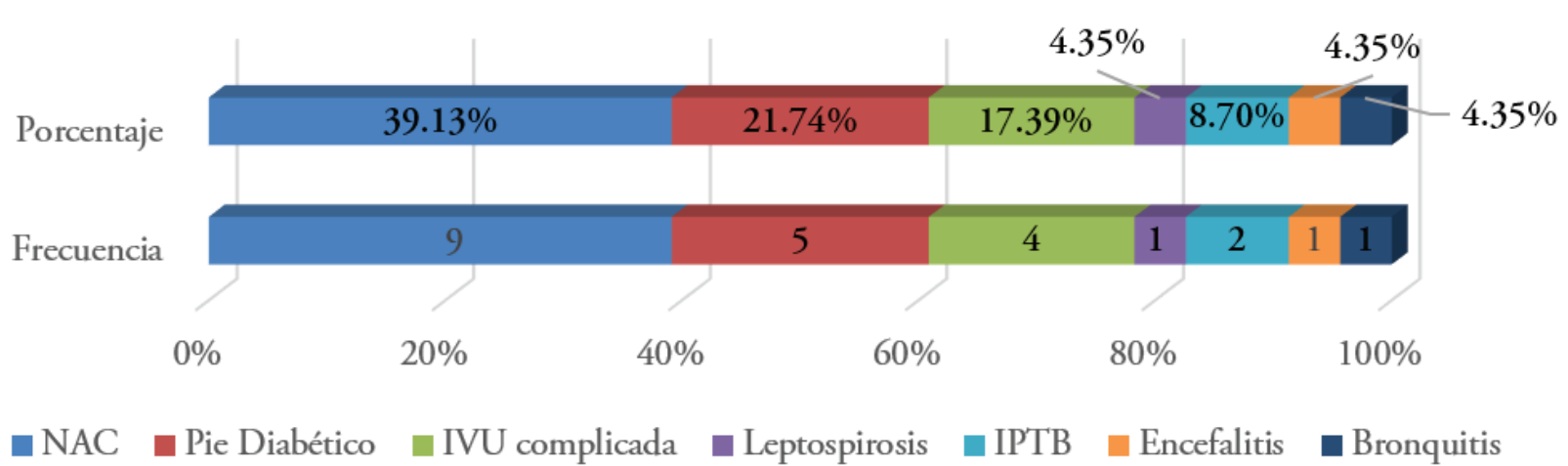

Figura 2. Caracterización del diagnóstico de ingreso de los pacientes con sepsis en el servicio de emergencia de Medicina Interna, Hospital Salvador Bienvenido Gautier, agosto-diciembre 2019.

Fuente: expedientes clínicos de los pacientes diagnosticados con sepsis
En la figura 2 se muestra la frecuencia de las principales causas de ingreso de los pacientes diagnosticados con sepsis. Se debe destacar que el 39.13\% corresponde a neumonía adquirida en la comunidad (NAC), seguido de pie diabético, con $21.74 \%$, e infección de vías urinarias (IVU) complicada, con $17.39 \%$

Las comorbilidades más frecuentes observadas en los pacientes con sepsis fueron diabetes mellitus e hipertensión arterial, presentándose cada una en un $65 \%$ de los casos, los cuales fallecieron en $53.33 \%$ y $46.67 \%$, respectivamente. El $30 \%$ presentaba enfermedad pulmonar obstructiva crónica (EPOC), fallecieron en $28.57 \%$ de los casos; se presentó cardiopatía en un $26 \%$, los cuales fallecieron en $83.33 \% ; 22 \%$ de enfermedad cerebrovascular (ECV), que fallecieron en $60 \%$; y $13 \%$ de otras comorbilidades. Solo un $4 \%$ de los pacientes que desarrollaron sepsis no presentaron comorbilidades conocidas.

El $78.26 \%$ presentaban más de dos comorbilidades concomitantes (véase figura 3 ).

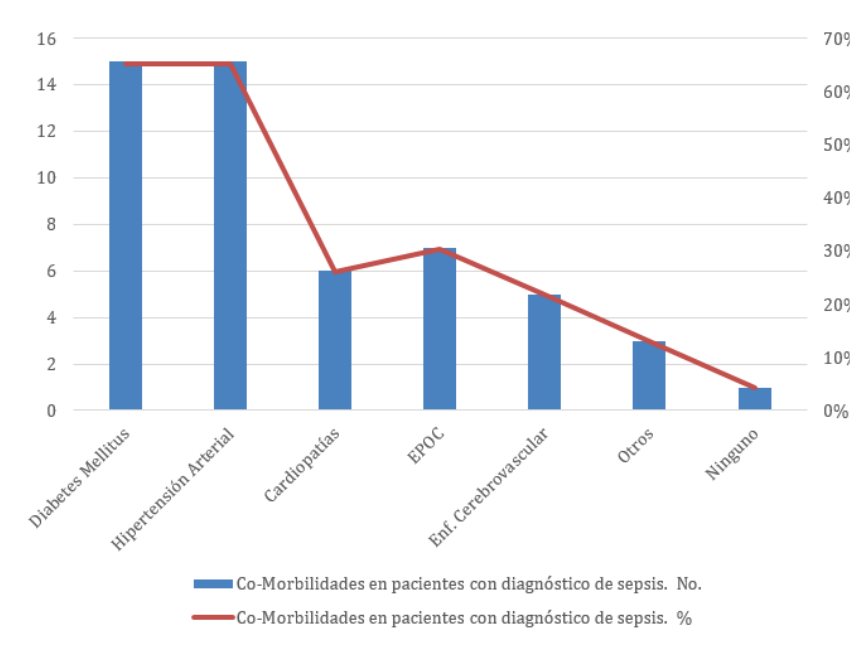

Figura 3. Comorbilidades identificadas en los pacientes diagnosticados con sepsis en el servicio de emergencia de Medicina Interna, Hospital Salvador Bienvenido Gautier, agosto-diciembre 2019

Fuente: expedientes clínicos de los pacientes con sepsis.

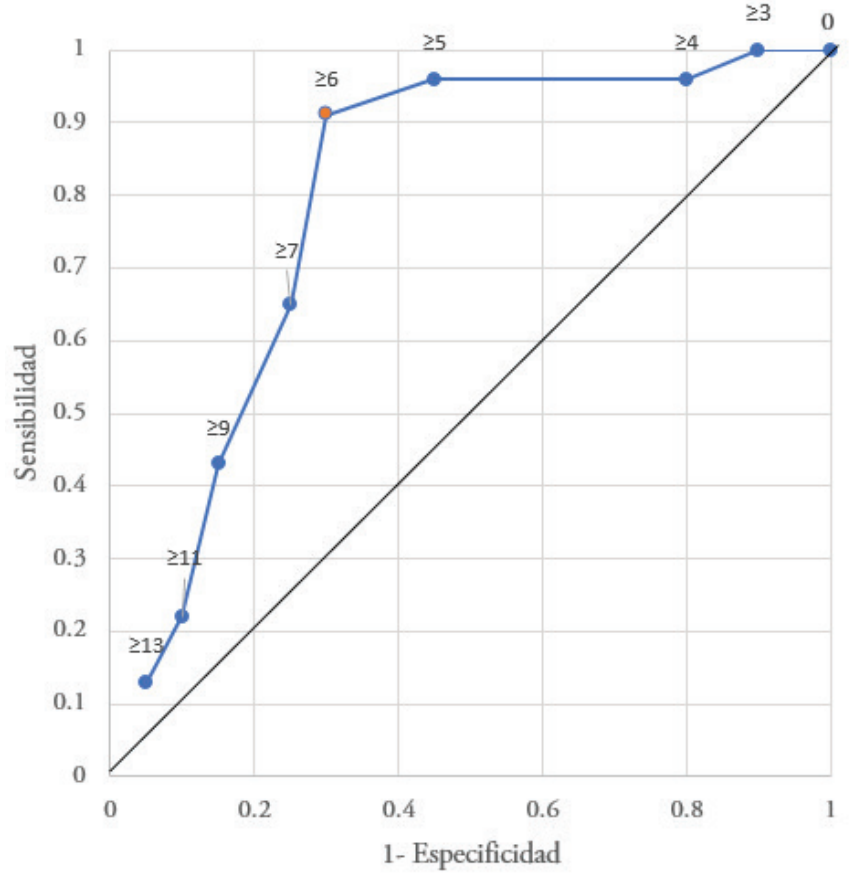

Figura 4. Gráfico ROC para escala NEWS en los pacientes con síndrome febril en el servicio de emergencia de Medicina Interna, Hospital Salvador Bienvenido Gautier, agosto-diciembre 2019.

Fuente: formularios completados con los expedientes clínicos de los pacientes diagnosticados con síndrome febril

Se presenta la curva ROC en la figura 4, obtenida para determinar la sensibilidad y especificidad de la escala NEWS en varios puntos de corte. Para el punto de corte $\geq 13$ se obtuvo una sensibilidad de $13 \%$ con una especificidad de $95 \%$. En el punto de corte $\geq 4$ se muestra una sensibilidad de $100 \%$ más una especificidad de solo $10 \%$. Se observa que el punto de corte $\geq 6$ de la escala NEWS obtuvo mejores valores simultáneos de sensibilidad (91\%) y especificidad (70 \%), presentando el mayor índice de Youden (0.61), valor predictivo positivo de $78 \%$, valor predictivo negativo de $88 \%$, razón de verosimilitud positiva y negativa, 3 y 0.1 , respectivamente. Cualquier valor superior de esta escala obtuvo mayor especificidad, pero, por tanto, la sensibilidad y valores predictivos caen rápidamente. El área bajo la curva fue de 0.84 (véanse tablas 2 y 3 ). 
Tabla 2. Estándar de referencia de la escala NEWS tomando como punto de corte la puntuación $\geq 6$ en los pacientes con síndrome febril

\begin{tabular}{|c|c|c|}
\hline NEWS & Sepsis & No sepsis \\
\hline+ & 21 & 6 \\
\hline- & 2 & 14 \\
\hline
\end{tabular}

Fuente: expedientes clínicos de los pacientes con síndrome febril.

Tabla 3. Características operativas de punto de corte de la escala NEWS $\geq 6$ en los pacientes con síndrome febril

\begin{tabular}{|l|l|}
\hline Punto de corte & $\geq \mathbf{6}$ \\
\hline Sensibilidad & $91 \%$ \\
\hline Especificidad & $70 \%$ \\
\hline Valor predictivo positivo & $78 \%$ \\
\hline Valor predictivo negativo & $88 \%$ \\
\hline Razón de verosimilitud positiva & 3 \\
\hline Razón de verosimilitud negativa 0.1 & \\
\hline
\end{tabular}

Fuente: expedientes clínicos de los pacientes con síndrome febril.

Tabla 4. Precisión de la escala NEWS como predictor de sepsis atendiendo al sexo en los pacientes con síndrome febril

\begin{tabular}{|c|c|c|c|c|c|c|c|}
\hline \multirow{2}{*}{ Sexo } & \multicolumn{2}{|c|}{ Bajo } & \multicolumn{2}{c|}{ Moderado } & \multicolumn{2}{|c|}{ Alto } & Total \\
\cline { 2 - 8 } & \multicolumn{2}{|c|}{ Sepsis } & \multicolumn{2}{|c|}{ Sepsis } & \multicolumn{2}{c|}{ Sepsis } & \\
\cline { 2 - 8 } & Si & No & Si & No & Si & No & \\
\hline Masculino & 0 & 4 & 4 & 3 & 10 & 1 & 22 \\
\hline Femenino & 2 & 6 & 3 & 1 & 4 & 5 & 21 \\
\hline Total & 2 & 10 & 7 & 4 & 14 & 6 & 43 \\
\hline
\end{tabular}

febril.

50 | Ciencia y Salud 2022; 6(1, enero-abril): 45-53 • Artículo origina
Tabla 5. Precisión de la escala NEWS como predictor de sepsis atendiendo a grupos de edad en los pacientes con síndrome febril

\begin{tabular}{|c|c|c|c|c|c|c|c|}
\hline \multirow{3}{*}{$\begin{array}{l}\text { Grupo } \\
\text { Etario }\end{array}$} & \multirow{2}{*}{\multicolumn{2}{|c|}{$\begin{array}{c}\text { Bajo } \\
\text { Sepsis }\end{array}$}} & \multirow{2}{*}{\multicolumn{2}{|c|}{$\begin{array}{c}\text { Moderado } \\
\text { Sepsis }\end{array}$}} & \multirow{2}{*}{\multicolumn{2}{|c|}{$\begin{array}{c}\text { Alto } \\
\text { Sepsis }\end{array}$}} & \multirow{3}{*}{ Total } \\
\hline & & & & & & & \\
\hline & $\mathrm{Si}$ & No & $S i$ & No & $S \mathbf{S i}$ & No & \\
\hline $18-30$ & 0 & 3 & 0 & 2 & 1 & 0 & 6 \\
\hline $31-40$ & 0 & 0 & 1 & 0 & 3 & 3 & 7 \\
\hline $41-50$ & 0 & 2 & 2 & 1 & 1 & 2 & 8 \\
\hline $51-60$ & 0 & 2 & 0 & 0 & 1 & 1 & 4 \\
\hline $61-70$ & 2 & 1 & 3 & 1 & 2 & 0 & 9 \\
\hline $71-80$ & 0 & 2 & 0 & 0 & 2 & 0 & 4 \\
\hline$>80$ & 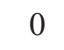 & 0 & 1 & 0 & 4 & 0 & 5 \\
\hline Total & 2 & 10 & 7 & 4 & 14 & 6 & 43 \\
\hline
\end{tabular}

Fuente: expedientes clínicos de los pacientes con síndrome febril.

Si aceptamos como punto de corte $\geq 6$ puntos en la escala NEWS para predecir el desarrollo de sepsis, tenemos que separar el grupo de moderado riesgo (5-6 puntos) determinando así que, en el sexo masculino, se obtuvieron 13 verdaderos positivos, 1 falso positivo, 1 falso negativo y 7 verdaderos negativos.

Lo que determinó una sensibilidad de $92.9 \%$ y especificidad de $87.5 \%$. Mientras que en el sexo femenino se obtuvieron 5 verdaderos positivos, 5 falsos positivos, 4 falsos negativos y 7 verdaderos negativos. Teniendo como resultado una sensibilidad de $55.6 \%$ y especificidad de $58.3 \%$ para el sexo femenino (véase tabla 4).

Como se muestra en la tabla 5, se pudo determinar que los rangos de edad en donde la escala NEWS fue mejor predictor de sepsis fueron de 18-30 y 71-80 años, con una sensibilidad y especificidad de $100 \%$ en ambos casos. Mientras que el grupo de 31-40 años alcanzó $100 \%$ de sensibilidad, la especificidad fue de $0 \%$. El rango de edad de 41-50 ańos obtuvo una sensibilidad de $100 \%$ y una espe- cificidad de $60 \%$. Para el grupo etario de 51-60 obtuvimos de sensibilidad y especificidad $100 \%$ y $66.7 \%$, respectivamente. En el rango de edad de 61-70 la sensibilidad fue de $42.9 \%$ y la especificidad de $100 \%$ El grupo mayor de 80 años logró una sensibilidad de $80 \%$

Tabla 6. Distribución de los casos según el riesgo de NEWS y la evolución clínica en los pacientes con síndrome febril

\begin{tabular}{|c|c|c|c|c|c|c|}
\hline \multirow{2}{*}{$\begin{array}{c}\text { Evolución } \\
\text { Clínica }\end{array}$} & \multicolumn{6}{|c|}{ Riesgo } \\
\cline { 2 - 7 } & \multicolumn{2}{|c|}{ Bajo } & \multicolumn{2}{c|}{ Moderado } & \multicolumn{2}{c|}{ Alto } \\
\cline { 2 - 7 } & No. & $\%$ & No. & $\%$ & No. & $\%$ \\
\hline Mejoría & 10 & $83.33 \%$ & 2 & $18.18 \%$ & 4 & $20 \%$ \\
\hline Monitoreo & 2 & $16.67 \%$ & 5 & $45.45 \%$ & 2 & $10 \%$ \\
\hline Muerte & 0 & $0 \%$ & 4 & $36.36 \%$ & 9 & $45 \%$ \\
\hline UCI & 0 & $0 \%$ & 0 & $0 \%$ & 5 & $25 \%$ \\
\hline Total & 12 & $100 \%$ & 11 & $100 \%$ & 20 & $100 \%$ \\
\hline
\end{tabular}

Fuente: formulario elaborado para la recolección de dato y expedientes clínicos de los pacientes con síndrome febril.

El $27.91 \%$ de todos los pacientes fue identificado por la escala NEWS como bajo riesgo, el $83.33 \%$ presentó mejoría clínica y el 16.67 \% necesitó monitoreo constante. Ningún paciente admitido en este estudio y clasificado por la escala como de bajo riesgo falleció o fue ingresado en UCI. De los pacientes de bajo riesgo solo 2 fueron diagnosticados con sepsis, representando el $16.67 \%$ de este grupo.

El grupo de riesgo moderado corresponde al $25.58 \%$ de todos los pacientes, de estos, el $45.45 \%$ necesitó de monitoreo constante, $36.36 \%$ falleció y el 18.18 \% restante mostró mejoría clínica. En este grupo, ninguno de los pacientes fue ingresado en UCI, 7 pacientes fueron diagnosticados con sepsis, lo que representa un $63.64 \%$.

De la población total, el $46.51 \%$ fue agrupado como de alto riesgo según la escala NEWS, donde encontramos que $45 \%$ de los pacientes en este grupo fallecieron, $25 \%$ necesitó de UCI, $20 \%$ presentó mejoría clínica y el $10 \%$ restante, monitoreo constante. El 70 \% desarrollaron sepsis (véase tabla 6).

\section{Discusión}

Con este estudio se buscó determinar la capacidad de la escala NEWS para predecir sepsis en el país, como ha sido detallado internacionalmente, logrando evidenciar una ceñida relación con los estudios previamente realizados a nivel internacional.

Se debe destacar que las causas principales de ingresos de los pacientes diagnosticados con sepsis fueron tanto la neumonía adquirida en la comunidad como las infecciones de vías urinarias complicada. Información que concuerda con el estudio realizado por Esper, et al. ${ }^{11}$, en el cual se determinó que el $33 \%$ de los casos de sepsis eran causados por infecciones respiratorias y $32 \%$ por infecciones genitourinarias.

En concordancia con nuestros hallazgos, Ynosencio, et al. ${ }^{12}$ describen las comorbilidades de los pacientes que sobrevivieron a sepsis en comparación con los que fallecieron, presentando hipertensión un $63 \%$ de los fallecidos y $59 \%$ los sobrevivientes, diabetes mellitus en $47 \%$ en los fallecidos y $30 \%$ de los sobrevivientes, enfermedad pulmonar en $32 \%$ de los fallecidos y $27 \%$ de sobrevivientes, enfermedad vascular en un $26 \%$ frente a un $21 \%$, mientras que en la enfermedad renal, $21 \%$ frente a $15 \%$.

Una puntuación en la escala NEWS mayor o igual a 6 obtuvo mejores valores simultáneos de sensibilidad y especificidad, a diferencia de lo que establecieron en la revisión de la escala NEWS en Inglaterra, en el 2017, por Royal College of Physicians $^{13}$, quienes seleccionaron como determinante de sepsis una puntuación mayor o igual a 5 .

Podemos contrastar los valores anteriormente mencionados con los obtenidos por el estudio realizado por Martín-Rodríguez et al. ${ }^{14}$ en España, en el 2019, donde mostraron que, utilizando una 
puntuación igual o mayor de 9 puntos, se observó una sensibilidad de $89 \%$, y una menor especificidad de $82 \%$, pero con un valor predictivo negativo de $99 \%$.

Sin embargo, Spagnolli et al. ${ }^{15}$, en el año 2017 , concluyeron que los pacientes que obtuvieron un valor de NEWS mayor a 4 en comparación a los pacientes que alcanzaron un valor menor de 4 , tuvieron un aumento en el riesgo de muerte aumentado, que iba de 12 a 36 veces.

\section{Conclusiones}

De un total de 43 pacientes con síndrome febril admitidos en el servicio de emergencia de medicina interna del Hospital Salvador Bienvenido Gautier en el período agosto-diciembre 2019, se pudo observar que una puntuación mayor o igual a 6 en la escala NEWS se presenta como un predictor estadísticamente significativo que ayudaría en el diagnóstico temprano de sepsis, siendo más sensible que especifica, lo que permite identificar a la mayor cantidad de pacientes con sepsis.

Las comorbilidades más frecuentes en los pacientes con sepsis fueron diabetes mellitus e hipertensión arterial, pero en las que se presentó mayor mortalidad fueron cardiopatías y enfermedad cerebrovascular, respectivamente. Más de la mitad de los pacientes que desarrollaron sepsis presentaban dos o más comorbilidades.

La escala NEWS fue mejor predictor de sepsis en el sexo masculino y en los grupos etarios de 18-30 y 71-80 años.

La neumonía adquirida en la comunidad (NAC), en la mayoría de los casos, fue el desencadenante de sepsis, seguido de Infección de vías urinarias (IVU) complicada y pie diabético.

De los pacientes identificados como bajo riesgo por la escala NEWS, una pequeña cantidad desarrolló sepsis, el resto presentó mejoría clínica sin necesitar atención medica constante. Poco menos de la mitad de los pacientes clasificados en riesgo moderado requirió de monitoreo médico constante, previo a su mejoría. La mayor cantidad de fallecidos y de diagnosticados finalmente con sepsis se encontraban dentro del grupo de alto riesgo.

\section{Referencias}

1. Singer M, Deutschman CS, Seymour CW, Shankar-Hari M, Annane D, Bauer M, et al. The Third International Consensus Definitions for Sepsis and Septic Shock (Sepsis-3). JAMA, 2016;315(8):801.

2. S. Munford, R. Septicemia y estado de choque séptico. En: Barnes PJ. Longo DL, Fauci AS, et al., (eds.). Harrison principios de medicina interna. Vol 2. $19^{a}$ ed. México: McGraw-Hill; 2015. p. 1751-1759.

3. Neira-Sánchez R, Málaga G. Sepsis-3 y las nuevas definiciones, jes tiempo de abandonar SIRS? Acta méd. Peru, [Internet]. 2016;33(3): 217-22. Disponible en: http://www.scielo.org. pe/scielo.php?script=sci_arttext\&pid=S172859172016000300008\&lng=es

4. Keeley A, Hine P, Nsutebu E. The recognition and management of sepsis and septic shock: a guide for non-intensivists. Postgraduate Medical Journal, 2017;93:626-34.

5. Bertullo M, Carbone N, Brandes M, Silva M, Meiss H, Tejera D et al. Epidemiología, diagnóstico y tratamiento de la sepsis severa en Uruguay: un estudio multicéntrico prospectivo. Rev. Méd. Urug, [Internet]. 2016;32(3):178-89. Disponible en:http://www.scielo.edu.uy/scielo.php?script $=$ sci_arttext\&pid $=S 1688039020160$ 00300007\&lng=es
6. Sinapidis D, Kosmas V, Vittoros V et al. Progression into sepsis: an individualized process varying by the interaction of comorbidities with the underlying infection. BMC Infect Dis, 2018;18(242). Available from https://doi.org/10.1186/s12879018-3156-z.

7. Faisal M, Richardson D, Scally AJ, Howes R, Beatson K, Speed K, Mohammed MA. Computer-aided National Early Warning Score to predict the risk of sepsis following emergency medical admission to hospital: a model development and external validation study. CMAJ, 2019;191(14):E382-E389. Doi: 10.1503/cmaj. 181418. PMID: 30962196; PMCID: PMC6 453675 .

8. Silcock DJ, Corfield AR, Gowens PA, Rooney KD. Validation of the National Early Warning Score in the prehospital setting. ELSAVIER [Internet]. 2015 [citado 8 enero 2022];89:31-35. Disponible en: https://www.sciencedirect.com/ science/article/abs/pii/S0300957215000118.

9. Usman OA, Usman AA, Ward MA. Comparison of SIRS, qSOFA, and NEWS for the early identification of sepsis in the Emergency Department. The American Journal of Emergency Medicine, 2019;37(8):1490-7.

10. Brink A, Alsma J, Verdonschot RJCG, Rood PPM, Zietse R, Lingsma HF et al. Predicting mortality in patients with suspected sepsis at the Emergency Department; A retrospective cohort study comparing qSOFA, SIRS and National Early Warning Score. Lopez-Delgado JC, (ed.). PLoS ONE. 2019;14(1):e0211133.
11. Esper A, Moss M, Lewis C et al. The role of infection and comorbidity: Factors that influence disparities in sepsis. Critica Care Medicine, 2014;34(10):2576-82. Doi:10.1097/01.CCM. $0000239114.50519 .0 \mathrm{E}$

12. Ynosencio T, Smith A, Endicott-Yazdani T et al. Co-morbidities in sepsis and sepsis related deaths. Critical Care Medicine, 2015;43(12):274. Doi: 10.1097/01.ccm.0000474920.02775.ee

13. Royal College of Physicians. National Early Warning Score (NEWS) 2: Standardising the assessment of acute-illness severity in the NHS. Updated report of a working party. London: RCP, 2017.

14. Martín-Rodríguez F, et al. Valor predictivo del preNEWS2-L (Pre-hospital National Early Warning Score 2 Lactate) para la detección de la mortalidad precoz en el ámbito prehospitalario. Emergencias, 2019;31:173-9.

15. Spagnolli W, Rigoni M, Torri E, Cozzio S, Vettorato E, Nollo G. Application of the National Early Warning Score (NEWS) as a stratification tool on admission in an Italian acute medical ward: A perspective study. Int J Clin Pract, 2017;71(3-4):e12934. 